\title{
Locutions en apprentissage de langue seconde
}

\author{
Dorota Sikora* \\ Université Littoral Côte-d'Opale, Centre du Musée, 34 Grande Rue, \\ BP 751, 62321 Boulogne-sur-Mer Cedex
}

\begin{abstract}
Résumé. L'article propose une réflexion à la fois linguistique et didactique sur les locutions et leur intégration dans l'enseignement de vocabulaire d'une langue étrangère. Les propriétés formelles, sémantiques et fonctionnelles de ces phrasèmes ont été largement décrites par la lexicologie, qui peut de ce fait apporter des indices précieux aux didacticiens. En nous appuyant sur la conceptualisation des locutions issue de la Lexicologie Explicative et Combinatoire, et en mettant à contribution leur modélisation en tant qu'unités lexicales dans la base de données Réseau Lexical du Français, nous nous interrogeons dans un premier temps sur les difficultés que les locutions présentent dans l'apprentissage de vocabulaire. Dans la seconde partie, nous proposons les grandes lignes d'une démarche pédagogique qui tient compte de ces propriétés linguistiques, tout en respectant le rythme de progression et d'acquisition de compétences défini par le Cadre européen commun de référence pour les langues (CECRL).
\end{abstract}

\begin{abstract}
The present paper addresses the issue of idioms in foreign language teaching. Their formal, semantic, and functional properties have been largely discussed and described in lexicology. Within the conceptual framework developed by Explanatory and Combinatorial Lexicology, and relying on the data encoded in French Lexical Network, the study firstly focalises on linguistic properties of idioms as main source of difficulties in language learning. The question that arises then is that of the possible methods of teaching idioms in a foreign language class. Thus, in a second part, the paper outlines a general design of an idiom pedagogy, in accordance with their linguistic properties on the one hand, and on the other, with acquisitional and organisational constraints of the language curricula defined in line with the Common European Framework of Reference for Languages.
\end{abstract}

\section{Introduction ${ }^{1}$}

Depuis l'élaboration des approches lexicalistes (cf. Nation 1982, 1990, Bogaards 1994 et surtout Lewis 1993, 2008) et le développement de la notion de phraséodidactique à partir des travaux de Gréciano (1994), il ne semble plus nécessaire de plaider en faveur d'une intégration des unités phraséologiques (désormais UP) dans un cursus de langue seconde (L2) ${ }^{2}$. On reconnaît en effet que le lexique d'une langue contient une multitude d'unités polylexicales de nature différente, qui se prêtent plus ou moins bien - souvent pas du tout - à un traitement décompositionnel. Elles doivent être saisies et apprises comme un

\footnotetext{
*Dorota.Sikora@univ-littoral.fr
} 
tout. La question qui se pose actuellement n'est donc pas s'il faut les enseigner, mais de savoir comment le faire de manière efficace.

La réflexion proposée ci-dessous poursuit un objectif bien pratique : il s'agit de montrer ce qu'une modélisation du lexique développée par des linguistes peut apporter à la didactique de vocabulaire, notamment à l'enseignement d'unités phraséologiques. Nous limitons la discussion aux locutions, non seulement parce que ce sont, comme le suggère Gläser (1988), des prototypes de tous les phraséologismes, mais surtout pour des raisons méthodologiques. Il est préférable, à notre avis, de ne pas aborder l'ensemble des UP, car les différents types de phrasèmes (locutions, collocations, pragmatèmes, proverbes, etc.) se caractérisent par des propriétés structurelles et fonctionnelles différentes. Par conséquent, les difficultés qu'ils posent aux apprenants et la façon de les traiter en classe ne sont pas les mêmes.

Les manuels de L2 semblent aujourd'hui quelque peu désarmés face aux locutions. Une étude réalisée par Berthemet (2017) montre qu'elles y sont très peu présentes : dix locutions à peine dans Version originale 1 (livre et cahier d'activités) contre 353 collocations. Elles y sont traitées en marge, souvent par un renvoi vers les dictionnaires. Or, comme nous aurons l'occasion de le voir dans la section 2, ces ressources n'offrent pas toujours aux apprenants l'assistance nécessaire. Partant de ce constat, nous discuterons des aspects didactiques, en nous appuyant sur des recherches expérimentales, menées sur plusieurs langues, qui revèlent et confirment certaines régularités et difficultés récurrentes dans l'apprentissage de vocabulaire (cf. Rodgers 1969, Laufer 1990, Nation 1990, Bogaards 1994, Milton 2009).

Le volet linguistique de la présente étude s'appuie sur une modélisation du lexique issue de la Lexicologie Explicative et Combinatoire (désormais la LEC), repensée dans le cours des travaux d'implémentation du Réseau Lexical du Français - RL-fr (Polguère 2014, 2016). Cette ressource offre aujourd'hui une description lexicographique de plus de 3200 locutions toute nature confondue, ce qui constitue un ensemble de données assez développé pour autoriser quelques généralisations. Chemin faisant, nous en adoptons également les conventions typographiques, en marquant les unités lexicales - lexèmes et locutions - en petites majuscules. En cas de polysémie, nous reprenons la numérotation des acceptions encodées dans le $R L-f r$.

La section qui suit propose une discussion des propriétés formelles et sémantiques des locutions en tant que sources de difficultés potentielles en apprentissage de vocabulaire. Dans la section 3, nous nous arrêtons sur les caractéristiques que l'on pourrait qualifier de fonctionnelles, voire interactionnelles, et sur le traitement que l'apprenant doit en assurer selon qu'il fait usage de ses compétences réceptives et productives. Dans la quatrième partie, avec un regard attentif sur les grilles de compétences du Cadre européen commun de référence pour les langues (CECRL 2001, North et Goodier 2018), nous réfléchirons sur les grandes lignes dont il faut tenir compte en construisant une démarche phraséodidactique visant tout particulièrement les locutions. La section 5 nous amènera à conclure que les lexicologues, les didacticiens et les enseignants ont tout intérêt à mieux se connaitre et à coopérer.

\section{Propriétés linguistiques des locutions et difficultés en apprentissage}

Si nous considérons, en suivant la LEC (cf. Mel'čuk et al. 1995, Mel'čuk 2013), que les locutions sont des unités lexicales (lexies) et par conséquent, des signes linguistiques, on pourra analyser leur structure selon les trois dimensions - formelle, sémantique et combinatoire - décrites par Mel'čuk et al. (1995). On sera ainsi en mesure de localiser avec précision les sources de difficultés que ce type de phrasèmes présente aux apprenants de L2. Dans cette section, nous nous intéresserons d'abord aux caractéristiques des signifiants locutionnels (2.1), puis à celles de leurs signifiés (2.2). Les propriétés combinatoires 
apparaîtront dans les deux sous-sections en rapport avec les facteurs de difficultés liés aux facettes formelle et sémantique des locutions.

\subsection{Structure syntagmatique des signifiants locutionnels}

La forme signifiante d'une locution est un syntagme composé d'au moins deux lexèmes reliés par des relations de dépendance. La figure 1 illustre le cas de FLEUR BLEUE, où FLEUR est gouverneur syntaxique de l'adjectif BLEU. Cette association d'un patron particulier, en l'occurrence $\mathrm{N}$ Adj, et de lexèmes qui le remplissent est appelée structure lexicosyntaxique ou SLS (cf. Pausé 2017).

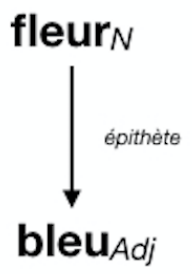

Fig. 1. Structure lexico-syntaxique de la locution FLEUR BLEUE.

Une SLS peut néanmoins être autrement plus complexe, notamment dans le cas de locutions verbales où un réseau de dépendances plus développé requiert, lors de la mise en discours, une solide maîtrise de la flexion verbale et un gestion appropriée de nombreux accords et de reprises anaphoriques. La figure 2 montre l'ensemble de relations entre les lexèmes qui forment la locution RENDRE LA MONNAIE DE SA PIÈCE. On voit dans l'exemple 1, extrait de la base textuelle Frantext, que son emploi en discours exige la maîtrise de la flexion verbale, ainsi que le recours aux possessifs et aux clitiques.

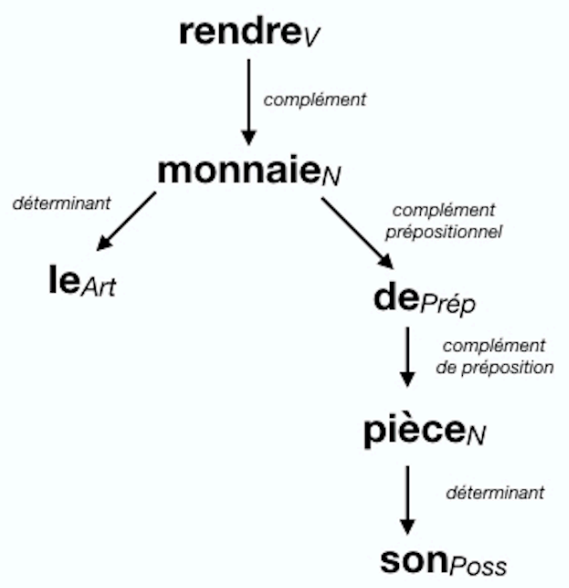

Fig. 2. Structure lexico-syntaxique de la locution RENDRE LA MONNAIE DE SA PIÈCE.

1. Elle a repris de l'aplomb, elle veut me rendre la monnaie de ma pièce. (Frantext)

La structure syntagmatique des signifiants locutionnels est source potentielle de trois types de difficultés souvent repérées dans l'apprentissage du vocabulaire. On observe en effet que la longueur d'une lexie, ainsi que sa partie du discours et ses caractéristiques flexionnelles facilitent ou, au contraire, constituent un obstacle pour la mémorisation et, par 
conséquent, pour son réemploi (cf. Laufer 1990). Nous verrons en 2.1.1 et en 2.1.2 comment une forme syntagmatique du signifiant conduit à renforcer ces facteurs de difficulté. Dans la sous-section 2.1.3, nous étudierons le décalage fréquent entre la nature d'une locution, c'est-à-dire sa partie du discours, et les fonctions syntaxiques qu'elle assume dans la phrase.

\subsubsection{Longueur}

Des tests réalisés sur plusieurs langues indiquent qu'il existe un rapport entre la longueur d'un lexème et la rapidité de son apprentissage. Plus un signifiant est long, plus son apprentissage serait difficile. En commentant ces résultats, Laufer (1990) souligne néanmoins que la longueur devient décisive au-delà d'un seuil défini en nombre de syllabes. Une certaine prudence s'impose dans la réflexion, tout d'abord parce que ces travaux portent essentiellement sur le vocabulaire lexémique, et non pas sur les expressions polylexicales. De plus, ils reposent sur la notion de mot, dont on connaît par ailleurs le caractère problématique. Néanmoins, on peut raisonnablement admettre que si la longueur d'un signifiant, mesurée en syllabes, est un facteur qui joue en faveur ou en défaveur d'une mémorisation rapide, elle est à prendre en compte tout autant pour les locutions que pour les lexèmes.

Une structure syntagmatique est, dans l'immense majorité des cas, plus longue qu'un lexème : des dissyllabes comme FLEUR BLEUE sont relativement rares. Or, la longueur a tendance à affecter la mémorisation et l'emploi en discours, notamment pour des raisons phonétiques. Les études réalisées auprès de plusieurs groupes d'apprenants (cf. par exemple : Meara 2009) montrent que les caractéristiques phonologiques et phonétiques jouent un rôle important pour un apprentissage efficace de vocabulaire en L2, tout particulièrement dans sa phase initiale. Une séquence aux caractéristiques articulatoires radicalement différentes de celles qu'on rencontre en L1 s'avère difficile à retenir et, par conséquent, à reproduire. Lorsqu'un apprenant prend la parole, une séquence polylexicale constitue un défi. Elle implique une série de sons à articuler plus complexe que celle d'un lexème et doit en plus s'inscrire dans un contour intonatif approprié.

\subsubsection{Complexité flexionnelle}

Lorsqu'on analyse les caractéristiques flexionnelles des locutions, il convient de distinguer deux cas de figure. Pour le premier, il n'est pas rare que les marques flexionnelles fassent partie du signifiant locutionnel. C'est le cas notamment de ATOMES CROCHUS, AVOIR LES CHEVILLES QUI ENFLENT, FAIRE DES GORGES CHAUDES et bien d'autres où les éléments de la SLS portent de manière permanente les exposants flexionnels ${ }^{3}$. Dans ce cas, l'apprenant n'a pas à prendre des décisions pour ce qui est de la flexion à appliquer; il s'agit des phénomènes de figement, dont nous parlerons plus amplement dans la sous-section 3.2.

Un second cas de figure doit être envisagé sur le plan pédagogique. Les relations de dépendance à l'intérieur du phrasème, qui se manifestent dans ses caractéristiques flexionnelles, peuvent requérir des décisions de la part de l'apprenant. Peut-on, doiton mettre une locution au pluriel, lorsqu'elle renvoie à plus d'un référent ? Si oui, faut-il fléchir tous les éléments lexicaux ou juste le pivot, c'est-à-dire le plus souvent, le gouverneur syntaxique ? Ainsi, dans le cas d'un syntagme nominal français FLEUR BLEUE, les marques du pluriel s'ajoutent aux deux lexèmes de la SLS. Ailleurs, par exemple pour POLITIQUE DE L'AUTRUCHE, ROND DE JAMBE, TEMPÊTE DANS UN VERRE D'EAU et bien d'autres, seul le gouverneur syntaxique est fléchi, alors que son complément reste invariable. Si le grammème ne se manifeste pas nécessairement à l'oral, le choix de déterminants et de modifieurs adjectivaux ne permet pas d'éviter ces questions. L'exemple 1 ci-dessus a déjà permis d'illustrer le maniement complexe des locutions 
verbales : dans un tel cas, la flexion s'accompagne d'un jeu de paramètres déictiques à organiser conformément à la situation d'énonciation.

On note ainsi un rapport entre la longueur d'un phrasème et ses propriétés flexionnelles : plus il y a de lexèmes dans sa SLS, plus les relations de dépendance sont complexes et la flexion compliquée.

\subsubsection{Partie du discours : entre nature et fonction}

Toute une série de travaux développés depuis plusieurs décennies (cf. Rodgers 1969, Allen et Valette 1972, Phillips 1981) tendent à montrer que, pour l'apprentissage des unités lexémiques, la partie du discours est un facteur susceptible de faciliter l'acquisition ou, au contraire, de la ralentir. Ainsi, les noms et les adjectifs semblent plus faciles à maîtriser que les verbes et les adverbes. Selon les langues et leurs systèmes flexionnels, ces résultats peuvent sans doute être affinés, mais il est certain qu'avec les locutions, la question devient particulièrement épineuse.

Pour l'illustrer, nous prendrons des exemples de locutions gouvernées par un nom, puisque cette partie du discours semble favoriser l'acquisition. De par leur nature, elles devraient être employées dans une phrase en position de sujet ou de complément. C'est effectivement ce qui arrive avec CHEVALIER SERVANT dans les exemples 2 et 3, employé respectivement dans ces deux fonctions.

2. Ton chevalier servant arrive ! (FrWac)

3. Enfin seule pour flirter tout mon soûl au téléphone avec le chevalier servant $d u$ moment. (Frantext)

La structure syntaxique de la locution FLEUR BLEUE est identique à celle de PRINCE CHARMANT. En surface, on lui attribue donc la même partie du discours : il s'agit d'une locution nominale. L'exemple 4 la montre cependant modifiée par l'adverbe LÉGĖREMENT et coordonnée avec un adjectif, assumant comme ce dernier une fonction d'épithète habituellement réservée aux adjectifs. Il en est ainsi de la locution nominale MAIN DANS LA MAIN dans l'exemple 5, où elle modifie le nom TRAVAIL, alors que dans 6, elle apparaît comme modificateur adverbial du verbe TRAVAILLER.

4. Cette aubaine s'expliquait en grande partie par l'affection maternelle et légèrement fleur bleue que madame Lamerise portait à son commis, au demeurant fort bon vendeur. (Frantext)

5. Je souhaiterais qu'il y ait un vrai travail main dans la main et plus d'échanges entre nous, explique-t-il. (Internet)

6. On travaille main dans la main avec la paroisse. (Internet)

Cela signifie que l'emploi de nombreuses locutions, tout particulièrement nominales et prépositionnelles, n'est pas celui que l'on pourrait anticiper à partir de leur partie du discours de surface. Ce décalage entre leur nature et leur fonction aboutit à des propriétés combinatoires difficilement prédictibles, ce qui ne facilite pas leur compréhension et encore moins leur réemploi dans le discours.

\subsection{Structure sémantique des locutions}

Les caractéristiques sémantiques d'un lexème ont une importance toute particulière pour l'apprentissage du vocabulaire, notamment en raison du rôle structurant que jouent les connexions paradigmatiques dans l'organisation de notre lexique mental (cf. Aitchison 2003). De nombreuses recherches montrent en effet que le vocabulaire d'un locuteur, que 
ce soit en L1 ou en L2 forme un réseau dont les nœuds sont reliés essentiellement par des relations de $\operatorname{sens}^{4}$ (cf. Meara et Wolter 2004, Schur 2007, Meara 2009). Or, certaines particularités sémantiques telles que le caractère abstrait d'un signifié ou sa faible granularité descriptive rendent l'apprentissage plus difficile. Dans cette section, nous nous proposons d'analyser les composants de la structure sémantique complexe que forment les signifiés locutionnels.

\subsubsection{Le sens dénotatif des locutions}

Tout comme les unités lexémiques, les locutions ont un signifié qu'on peut expliciter dans une paraphrase définitoire. Ainsi, pour expliquer le sens de la locution prépositionnelle AUX ANGES, ${ }^{5}$ on pourra proposer la définition suivante :

\section{AUX ANGES = 'qui éprouve un sentiment de joie intense et une sensation de bien-être'}

En tant que lexie pourvue d'un sens dénotatif ou descriptif (cf. Kleiber 1990, 1999), une locution s'inscrit ainsi dans le vaste réseau que forme le lexique, en tissant des relations avec d'autres lexies mono- ou polylexicales. La figure 3 ci-dessous montre le sous-graphe, contruit à partir des données encodées dans la base lexicale $R L-f r$, organisé autour de AUX ANGES. Cette lexie est reliée par des relations de quasi-synonymie aux adjectifs RAVI, HEUREUX, CONTENT, etc., ainsi qu'à une autre locution prépositionnelle SUR UN NUAGE. Ces connexions permettent également de connecter AUX ANGES, quoique indirectement, à son quasi-antonyme MALHEUREUX et d'établir les liens avec des lexies nominales telles que BONHEUR, CONTENTEMENT, FÉLICITÉ et SEPTIÈME CIEL, puis avec leur hyperonyme commun SENTIMENT1a ${ }^{6}$. Les couleurs permettent d'identifier des groupements de nœuds (clusters) fortement interconnectés.

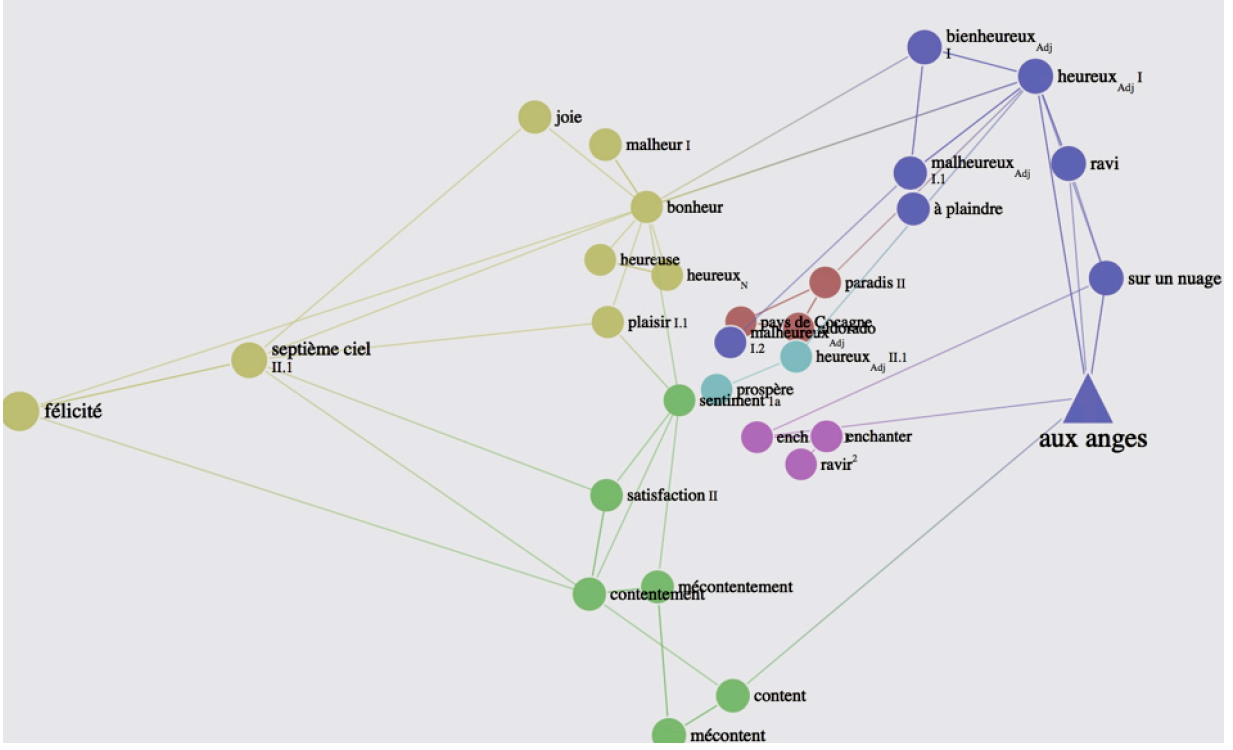

Fig. 3. Réseau de relations paradigmatiques de la locution prépositionnelle AUX ANGES (extrait de la base de données lexicales $R L-f r$.

En s'appuyant sur les paraphrases définitoires, on pourra qualifier le sens dénotatif des locutions de non-compositionnel, puisque leur sens global n'est pas le résultat d'un calcul 
effectué sur les sens des lexèmes qui forment sa $\operatorname{SLS}^{7}$. Nous verrons plus loin (section 3.1) en quoi la non-compositionnalité d'un signifié locutionnel constitue un défi dans l'apprentissage d'une L2.

Certaines locutions - HÔTESSE DE L'AIR, ROUGE À LÈVRES, SALLE DE BAINS, SIÈGE SOCIAL - ont un sens dénotatif concret que l'on peut rendre précisément dans une paraphrase. Cependant, dans de nombreux cas, il n'en est pas ainsi. Comme le souligne Fellbaum $^{8}$, les locutions sont là entre autres pour nous permettre de décrire des situations complexes d'une manière courte et condensée. Elles permettent de nommer, voire de qualifier, une grande diversité de situations. Pour ce faire, leur sens contient nécessairement une bonne dose de vague, qui se traduit par des paraphrases définitoires très générales. Elles s'avèrent par conséquent difficilement accessibles, surtout pour un apprenant.

$\mathrm{Ci}$-dessous, dans les figures 4 et 5 , nous reproduisons les définitions de PEAU DE BANANE proposées dans le Petit Robert électronique et dans le Trésor de la Langue française informatisé (désormais $T L F i)^{9}$. Elles recourent respectivement aux lexèmes PROCÉDÉ et $\mathrm{CE}$, qui autorisent tous les deux une multitude de référents de nature très diverse. De plus, la paraphrase du Petit Robert contient une expression placée entre guillemets, signalant sans plus de précision qu'elle est employée de manière inhabituelle.

- Loc. fig. Peau de banane : procédé déloyal destiné à « faire tomber » qqn. Glisser une peau de banane à qqn (cf. Savonner* la planche à qqn).

Fig. 4. Description lexicographique de la locution PEAU DE BANANE dans le Petit Robert électronique.

- Au fig. Peau de banane. Ce qui fait tomber :

-3. ... il y a trop d'embâches, trop de gens sur votre chemin qui ont intérêt à glisser sous vos pas la peau de banane... P. VIALAR, La Mort est un commencement, La Carambouille, 1949, p. 166.

Fig. 5. Description lexicographique de la locution PEAU DE BANANE dans le TLFi.

Les didacticiens savent qu'une lexie au sens abstrait est plus difficile à apprendre que celle qui dénote une entité concrète (Laufer 1990). Qu'en est-il d'une unité au sémantisme vague ? Le cadre de cet article ne permet pas de discuter en détail le rapport entre l'abstrait et le vague; ainsi, nous limiterons-nous à souligner simplement que, malgré certains recoupements, il ne s'agit aucunement d'équivalence. Selon Mel'čuk et al. (1995), un sens est vague, lorsqu'il correspond à plus d'un référent extralinguistique ou plutôt, dirons-nous, à plus d'une catégorie de référents extralinguistiques ${ }^{10}$. En effet, PEAU DE BANANE peut renvoyer à des faits aussi variés qu'une question mal intentionnée, une visite inopportune ou toute action destinée à mettre quelqu'un dans une situation délicate. La locution VAGUE À L'ÂME, au contraire, est bien une lexie abstraite, puisque sa dénotation est construite en intension à l'aide d'un ensemble de traits sémantiques, mais elle n'est pas vague sur le plan référentiel. Elle sert à nommer une émotion bien particulière.

Le sens dénotatif, paraphrasable tant bien que mal dans une définition lexicographique, joue un double rôle. D'une part sur le plan référentiel, il permet de circonscrire un ensemble de situations auxquelles la locution s'applique. De l'autre, il sert à établir des connexions paradigmatiques avec d'autres unités lexicales (cf. la figure 3 ci-dessus). Les difficultés que pose l'acquisition des lexies abstraites et vagues tiennent certainement à 
plusieurs raisons; notre explication se veut purement lexicale et ne peut, de ce fait, prétendre à exhaustivité.

Les lexies abstraites sont difficiles à retenir, car l'ensemble de traits définitoires en intension qu'il faut maîtriser, par exemple pour VAGUE À L'ÂME, doit ensuite être mis en relation avec un autre ensemble de traits définitoires, par exemple celui de TRISTESSE. Une lexie vague comme PEAU DE BANANE est difficile à retenir, puisqu'elle renvoie à des ensembles de référents très divers (CE ? PROCÉDÉ ?) dont parfois seul le locuteur perçoit des caractéristiques correspondant au sens lexical. La granularité descriptive d'une lexie vague la situe bien au-dessus de niveau de base, celui qui offre l'image d'un prototype du référent (cf. Rosch 1975, Kleiber 1990, Taylor 1995).

Ainsi, le sens dénotatif de plusieurs locutions - abstrait et/ou vague - rend leur acquisition particulièrement complexe, même si par ailleurs les lexicographes parviennent à en formuler, tant bien que mal, une définition.

Cependant, même en présence d'une définition explicite du sens dénotatif, une autre dimension sémantique doit souvent être prise en compte. La locution verbale COUPER LES CHEVEUX EN QUATRE signifie 'examiner une question avec une attention excessive pour des détails sans importance', ce qui la positionne dans le réseau lexical comme quasi-synonyme de CHERCHER LA PETITE BÊTE, des lexèmes PINAILLER, CHIPOTER, ainsi que, vulgairement, de ENCULER LES MOUCHES EN PLEIN VOL. Comme pour de nombreuses autres lexies locutionnelles, pour saisir sa particularité, il est, semble-t-il, nécessaire d'aller au-delà du sens dénotatif.

\subsubsection{Sens figuratif ou figuré ?}

Lors d'un cours de FLE, nous avons été amenée à expliquer aux étudiants de niveau A2 le sens de la locution nominale PEAU DE BANANE, tel qu'il est illustré dans l'exemple 7 cidessous :

7. Une petite peau de banane par-ci, un croc-en-jambe par-là, une méchanceté bénigne et un café offert pour sceller tout ça, vas y on est pote, la vie est belle. (Internet)

Contexte aidant, les étudiants sont parvenus à inférer le sens dénotatif de PEAU DE BANANE : 'situation défavorable mise en place, de manière sournoise, par X contre Y pour faire échouer les projets de ce dernier', mais nos tentatives - certainement maladroites - de compléter la caractérisation sémantique de cette locution par une description de la métaphore et de l'image qu'elle véhicule (celle d'un individu, qui tout en avançant marche sur une véritable peau de banane, glisse et tombe) ont semé la confusion. Or, sans cette charge imagée, la connaissance de cette lexie locutionnelle ne peut pas être considérée comme complète.

Plusieurs travaux (cf. Gibbs 1994, Dobrovol'skij et Piirainen 2005, Piirainen, 2008a, Dobrovol'skij 2016 à titre d'exemple) soulignent que les locutions, tout comme les proverbes et bien d'autres unités polylexicales, font partie de ce que l'on dénomme en anglais figurative language. C'est à dessein que nous nous arrêtons sur cette notion dans sa forme anglophone. L'adjectif FIGURATIVE souvent employé dans ces travaux pour décrire le sémantisme des locutions est polysémique, avec deux acceptions susceptibles d'interférer, voire de prêter à confusion. Pris dans un premier sens, FIGURATIVE I peut être paraphrasé par 'used with a non basic meaning' et traduit en français par l'adjectif FIGURÉ, opposé à LITTÉRAL. Dans une seconde acception, FIGURATIVE II est un cognat du qualificatif français FIGURATIF, que nous paraphraserons simplement ici comme 'relatif à la représentation ou à l'image d'un objet' ${ }^{11}$. Pour éviter tout malentendu, il convient d'expliquer le lien entre le caractère figuratif et figuré du sens locutionnel. 


\section{Un sens figuratif ?}

Une locution telle que COUPER LES CHEVEUX EN QUATRE a, nous l'avons dit plus haut, un sens dénotatif. Cependant, la paraphrase qui en a été proposée semble incomplète. En effet, elle ne rend pas compte de la répresentation qui surgit lorsque le sens dénotatif est actualisé dans un énoncé : celle d'un individu qui s'applique à séparer en plusieurs parties des objets aussi fins et insignifiants que des cheveux, tâche dont l'utilité reste difficile à établir. De même, en définissant PEAU DE BANANE (cf. l'exemple 7 ci-dessus) comme 'situation défavorable mise en place, de manière sournoise, par X contre $\mathrm{Y}$ pour faire échouer les projets de ce dernier', on ne mentionne d'aucune façon l'image qui accompagne le sens véhiculé.

Ainsi, on pourra admettre que le sens d'une locution est figuratif en ce qu'il véhicule une composante imagée. Selon Dobrovol'skij (2016), une composante imagée (cf. ibidem, the image component) est faite de traces linguistiquement pertinentes de l'image sousjacente $^{12}$. Il en ressort - comme souligné par l'auteur - que cette composante est bien plus qu'une représentation mentale. Elle est linguistiquement pertinente dans la mesure où elle détermine, du moins en partie, la combinatoire lexicale et les restrictions d'emploi.

La composante imagée se laisse en effet détecter dans le sémantisme de PEAU DE BANANE sur plusieurs plans. On la repère par la présence d'un premier actant $\mathrm{X}$ ou encore par la composante d'une manière sournoise dans la paraphrase définitionnelle. Ces éléments sémantiques peuvent être mis en relation avec une préférence nette que montre cette locution pour le collocatif verbal GLISSER III.2 qui avec une structure argumentale à trois places $(\mathrm{X} \sim \mathrm{Y}$ à $\mathrm{Z}$, où la position actancielle $\mathrm{Y}$ est remplie par PEAU DE BANANE) exprime la causation, et non pas le sens de base 'perdre l'équilibre en se déplaçant sur une surface lisse' (X $\sim$ sur $\mathrm{Y})$.

La définion de composante imagée proposée par Dobrovol'skij (2016) est plus restreinte que l'image que nous avons tenté de décrire pour COUPER LES CHEUVEUX EN QUATRE et PEAU DE BANANE, car elle n'inclut que les éléments repérables dans le sens dénotatif. En nous interrogeant sur leurs origines, nous devons à présent nous tourner vers ce qui, en sémantique des locutions, relève du figuré tout autant que du figuratif.

\section{Un sens figuré ?}

Certains travaux (Gibbs 1994) laissent penser que l'adjectif FIGURATIVE devrait être traduit par FIGURÉ pour caractériser le sens dénotatif des locutions par opposition à un sens littéral. Cependant, analyser le sémantisme d'une locution en le considérant comme figuré semble quelque peu problématique pour deux raisons. Premièrement, parce que cela laisse penser qu'une locution a d'abord un sens littéral. Si tel était le cas, pourrait-on encore parler d'une locution?

Deuxièmement, notre approche s'appuie sur un modèle lexical en synchronie, avec des prérequis théoriques et méthodologiques bien précis. Nous nous intéressons aux locutions en tant qu'unités lexicales, donc en tant qu'entités constitutives du lexique français telles qu'elles se présentent aux locuteurs contemporains. Or, qualifier leur sens dénotatif de figuré nous conduirait à postuler l'existence de phrasèmes au statut lexical difficile à déterminer: ce ne serait pas des locutions, puisqu'elles auraient un sens littéral, donc potentiellement compositionnel. Aussi bien une modélisation lexicographique que le traitement didactique d'un tel construit seraient à notre avis hautement problématiques, notamment parce que loin de caractériser le sens lexical, ils s'appliqueraient plutôt aux mécanismes qui produisent des contenus imagés, c'est-à-dire figuratifs.

Il est probable, comme le suggèrent de nombreux auteurs (cf. Gibbs 1994, Dobrovol'skij et Piirainen 2005 à titre d'exemple), que le sens dénotatif d'une locution soit effectivement une métaphore ou une métonymie lexicalisée, résultat de mécanismes cognitifs omniprésents dans la pensée et dans la langue, responsables entre autres d'extensions polysémiques dans le cas d'unités monolexémiques. Plutôt que de le qualifier de figuré et, par conséquent, de le considérer comme dérivé d'un sens littéral, nous le 
traitons comme donnée première en synchronie: une locution peut avoir un caractère figuratif, lié entre autres à la présence d'une composante imagée telle que définie par Dobrovol'skij (2016). Dans la sous-section suivante, nous proposons une approche de contenus figuratifs des locutions à travers leur SLS.

\section{Analyser les contenus figuratifs de locutions}

Le sens dénotatif d'une locution est figuratif en ce qu'il contient des traces linguistiquement pertinentes de l'image sous-jacente, qui forment sa composante imagée. Leur pertinence linguistique peut se manifester par exemple sur le plan combinatoire (cf. Dobrovol'skij 2016) ou bien dans le registre : ENCULER LES MOUCHES EN PLEIN VOL se laisse paraphraser par 'attacher une importance excessive aux détails insignifiants', définition qui ne justifie en rien la marque d'usage vulgaire. Celle-ci est due au lexème verbal, dont le sémantisme est pourtant absent de la paraphrase définitoire.

La notion de strucure lexico-syntaxique, SLS (cf. section 2.1 ci-dessus), permet en effet d'établir des liens entre la composante imagée du sens d'une locution et les unités qui la constituent en tant que syntagme. Si la composante imagée est bien présente, c'est en raison d'une mise en relation avec le syntagme libre à la SLS identique. ${ }^{13}$ Autrement dit, le contenu figuratif des locutions telles que PEAU DE BANANE et COUPER LES CHEVEUX EN QUATRE est à lier avec les syntagmes PEAU II DE I BANANE I et COUPER I.1a LES CHEVEUX I EN IV.3 QUATRE 1b. Leurs SLS sont construites de manière identique, mais en numérotant les lexies, nous indiquons l'apport sémantique de chaque élément lexical. Cet apport peut être relativement complexe, différent d'une locution à l'autre. Ci-dessous, les tableaux 1 et 2 permettent de comparer le cas de PEAU DE BANANE et celui de COUPER LES CHEVEUX EN QUATRE.

\section{Tableau 1 : Collocation et locution (PEAU DE BANANE)}

\begin{tabular}{|c|c|}
\hline PEAU II DE I BANANE I (syntagme libre) & PEAU DE BANANE (locution) \\
\hline $\begin{array}{l}\text { PEAU II - 'enveloppe extérieure d'un fruit' } \\
\text { BANANE I - 'fruit de forme allongée et } \\
\text { légèrement courbée dont la peau a des } \\
\text { propriétés glissantes' } \\
\text { - La peau de banane a des propriétés } \\
\text { physiques dangereuses pour celui qui } \\
\text { marche dessus par inadvertance. } \\
\text { - La banane est un fruit considéré } \\
\text { comme particulièrement apprécié des } \\
\text { singes. } \\
\text { Les singes sont considérés comme } \\
\text { des animaux agiles et ingénieux, } \\
\text { connus pour leurs tours de malice. }\end{array}$ & $\begin{array}{l}\text { PEAU DE BANANE - 'situation défavorable } \\
\text { mise en place, de manière sournoise, par } \\
\text { X contre Y, pour faire échouer les projets } \\
\text { de ce dernier' } \\
\text { - Situation dangereuse, susceptible } \\
\text { de déstabliser Y. } \\
\text { Xagit d'une manière agile et } \\
\text { ingénieuse, non dépourvue de } \\
\text { malice. }\end{array}$ \\
\hline
\end{tabular}

Dans la colonne de gauche (syntagme libre), en italiques, apparaissent des composantes relevant à la fois du sens de BANANE et des connotations liées non seulement à cette lexie, mais également au lexème SINGE qui n'apparaît pas explicitement ${ }^{14}$. La pertinence linguistique de cette référence simiesque se manifeste dans le collocatif GLISSER : glisser quelque chose à quelqu'un suppose en effet une certaine agilité, qu'on reconnaît habituellement aux singes justement.

Le cas de COUPER LES CHEVEUX EN QUATRE semble plus complexe encore en ce que le syntagme libre offre potentiellement beaucoup plus d'éléments que n'en contient la composante imagée de la locution. De nouveau, nous les réunissons dans la colonne de 
gauche du tableau 2, en sélectionnant à droite ceux qui forment la composante imagée de la locution.

Tableau 2 : Collocation et locution (COUPER LES CHEVEUX EN QUATRE)

\begin{tabular}{|c|c|}
\hline $\begin{array}{l}\text { COUPER I.1a LES CHEVEUX I EN IV.3 } \\
\text { QUATRE } 1 \mathrm{~b} \text { (syntagme libre) }\end{array}$ & $\begin{array}{c}\begin{array}{c}\text { COUPER LES CHEVEUX } \\
\text { (locution) }\end{array} \\
\text { (lon QUATRE } \\
\end{array}$ \\
\hline $\begin{array}{l}\text { COUPER I.1a - 'séparer Y en plusieurs } \\
\text { petites parties à l'aide d'un instrument } \\
\text { tranchant' } \\
\text { CHEVEU I - 'poil qui recouvre la tête' } \\
\text { - Un cheveu un élément du corps très } \\
\text { fin, délicat et fragile. Pris à l'unité, il } \\
\text { est à peine visible. } \\
\text { Couper sélectionne un argument } \\
\text { Instrument, dont l'emploi n'est pas } \\
\text { indispensable pour un Y aussi délicat } \\
\text { qu'un cheveu. } \\
\text { Couper un cheveu (en quatre) conduit } \\
\text { à diviser quelque chose de fin, fragile } \\
\text { et insignifiant, en créant des parties } \\
\text { encore plus fines et encore plus } \\
\text { insignifiantes. } \\
\text { C'est un travail inutile. } \\
\text { Couper un objet fin en plusieurs } \\
\text { parties encore plus fines est un travail } \\
\text { long et délicat. } \\
\text { Un tel travail repose sur } \\
\text { l'application, la précision et la } \\
\text { méticulosité de l'individu qui le } \\
\text { réalise. }\end{array}$ & $\begin{array}{l}\text { COUPER LES CHEVEUX EN QUATRE - } \\
\text { 'examiner une question avec une attention } \\
\text { excessive aux détails dépourvus } \\
\text { d'importance' } \\
\text { - travail inutile et dépourvu d'intérêt } \\
\text { - perte de temps } \\
\text { - agent X présenté sous un jour } \\
\text { défavorable de pinailleur }\end{array}$ \\
\hline
\end{tabular}

Les éléments sémantiques réunis dans la colonne de gauche - composantes de sens lexical, implications conventionnelles (entailments) et contextuelles - sont nettement plus nombreuses que les traces linguistiquement pertinentes repérables à droite. En effet, la scène décrite par le syntagme libre forme une image suffisamment riche pour nourrir potentiellement deux types de contenus sémantiques différents : l'un positif, car mettant en avant les qualités telles que soin et précision apportés au travail, et l'autre, réellement lexicalisé, tendant vers le pôle négatif avec l'idée d'un entêtement inutile.

\subsection{Propriétés linguistiques des locutions et difficultés d'apprentissage : un bilan}

Cette section a permis de passer en revue les propriétés qui caractérisent les locutions en tant qu'unités lexicales, c'est-à-dire en tant qu'unités constitutives du lexique. D'un point de vue formel, ce sont des syntagmes aux structures plus ou moins complexes. De même, leur sens ne se limite pas, dans une bonne partie des cas, à la dimension dénotative qui, par ailleurs, peut être abstraite ou vague. Bien que non compositionnel en synchronie, il maintient souvent un lien avec les lexèmes de sa SLS. Les locutions semblent cumuler les propriétés qui sont des sources nombreuses de difficultés connues dans l'apprentissage de vocabulaire. Pour les maîtriser, un apprenant de L2 doit s'approprier une masse 
d'informations de nature très différente. Cependant, comme nous le verrons dans la section 3 , ces connaissances - relatives toujours à une locution particulière plutôt qu'à tous les phrasèmes de ce type - doivent être complétées par des compétences appropriées.

\section{Fonctionnement linguistique des locutions et compétences d'apprenants}

Pour décrire les locutions, on recourt aux notions de figement, de (non-) compositionnalité, de (non-) analysabilité, de transparence et d'opacité, de motivation. Si les définitions de ces concepts sont loin de faire l'unanimité dans la communauté des phraséologues, c'est parce que, nous semble-t-il, elles décrivent des aspects différents, souvent complémentaires, du fonctionnement sémantique et discursif des locutions. C'est d'ailleurs la raison pour laquelle il est si intéressant, à notre avis, d'étudier ces notions dans un contexte didactique. Dans cette section, notre objectif est de montrer dans quelle mesure il convient de les prendre en compte dans l'acquisition de différentes compétences en L2. Celles-ci, quel que soit le niveau auquel le CECRL (2001, North et Goodier 2018) les assigne, se répartissent en compétences réceptives et compétences productives ${ }^{15}$.

\subsection{Locutions et compétences réceptives de l'apprenant}

Les compétences réceptives en langue étrangère reposent à la fois sur des connaissances déclaratives et procédurales que l'apprenant doit mobiliser pour comprendre un message oral ou écrit, et sur une démarche interprétative qui lui permettra de faire un usage approprié de ces connaissances pour mettre en place des inférences et des devinements face à des éléments implicites ou simplement inconnus. La première tâche qu'il doit mener à bien consiste à décomposer des segments du discours pour isoler les éléments significatifs et leurs interactions tant sur le plan sémantique que pragmatique.

On pourrait difficilement envisager l'apprentissage d'une langue autrement qu'à partir de la compréhension: l'étudiant est en effet amené à donner une interprétation à un segment de discours, à le décomposer pour identifier les unités significatives. Par exemple, face à la locution PEAU DE BANANE dans l'énoncé (7), une première étape consiste à l'identifier comme un signifiant polylexical. Soit il s'agit d'une lexie déjà connue, soit l'étudiant doit partir à la découverte d'un sens inconnu. Il dispose, pour ce faire, d'un certain nombre d'indices co(n)textuels et lexico-syntaxiques.

Dans sa démarche interprétative, il est possible que, dans un premier temps, notre apprenant procède à une décomposition en $\mathrm{N}_{1}$ (PEAU II 'enveloppe externe d'un fruit'), Prép (DE I), $\mathrm{N}_{2}$ (BANANE 'fruit à chair compacte, de forme allongée et courbée, avec une enveloppe jaune'). Or, en raison de la non-compositionnalité du sens dénotatif, le résultat d'un calcul effectué à partir de ces lexèmes s'accorde mal avec les indications cotextuelles (CROC-EN-JAMBE, MÉCHANCETÉ, CAFÉ) conduisant vers un autre sens potentiellement actualisé dans (7). Autrement dit, une réanalyse s'impose pour formuler une autre hypothèse.

7. Une petite peau de banane par-ci, un croc-en-jambe par-là, une méchanceté bénigne et un café offert pour sceller tout ça, vas y on est pote, la vie est belle. (Internet)

Dans (7), PEAU DE BANANE fait partie d'une coordination avec les lexèmes CROC-ENJAMBE et MÉCHANCETÉ II, permettant d'identifier le champ sémantique d'attitudes et de comportements dont relève la locution. Un raisonnement analogique pourra conduire même à une structure argumentale similaire : tout comme CROC-EN-JAMBE et MÉCHANCETÉ II, 
PEAU DE BANANE engage deux participants (celui qui la glisse et celui qui en subit les conséquences).

Un deuxième type d'indices à explorer s'offrent à travers la SLS de la locution. En poursuivant la recherche de signification, il convient d'observer les lexèmes qui forment le syntagme libre PEAU II DE I BANANE I. Il s'agit d'une réanalyse susceptible d'amener l'apprenant à une certaine propriété des peaux de banane qui les rend glissantes et, de ce fait, dangereuses pour l'équilibre et l'intégrité physique de quiconque marche dessus. Le succès de cette opération dépend aussi bien du vocabulaire dont on dispose que des connaissances extra-linguistiques et des inférences que l'on est en mesure de construire.

En ajustant la définition proposée par Langlotz (2006), on pourra dire qu'une locution est analysable, lorsque, à travers les contenus sémantiques ${ }^{16}$ associés aux éléments lexicaux qui la forment, on est en mesure d'identifier son sens. L'analysabilité n'est donc pas une propriété de l'unité lexicale elle-même, mais plutôt une caractéristique interactionnelle : elle tient entièrement à celui ou celle qui procède à l'analyse. En conséquence, la locution peut devenir transparente ; comme l'explique Svensson (2008: 84) : “when presented with an expression, a language user understands it without any problems, without any other previous knowledge than understanding the separate words that make up the expression, then it is transparent."

L'analyse réalisée par le locuteur apprenant permet non seulement de construire une interprétation et, chemin faisant de comprendre le sens dénotatif de la locution, mais également de la motiver, c'est-à-dire d'établir le ou les liens qui connectent son sens à la signification de la collocation ou du syntagme libre correspondants. On pourra ainsi admettre que si une locution est sémantiquement transparente, elle devient motivée. Mais une fois de plus, il s'agit alors non pas de propriété inhérente des lexies locutionnelles, mais d'une caractéristique interactionnelle. On verra ci-dessous que la transparence est parfois trompeuse et conséquemment, la motivation caduque.

Les indices dont disposent les locuteurs apprenants sont parfois extrêmement minces. Dans une série de tests, deux groupes d'étudiants de Master Didactique des Langues, locuteurs natifs du français d'une part, étudiants étrangers de niveau B2 de l'autre, ont dû examiner une liste de treize locutions parmi lesquelles figurait TEMPÊTE DANS UN VERRE D'EAU. Dans un premier temps, elles leur ont été présentées de manière entièrement décontextualisée pour vérifier s'ils les connaissaient et, en cas de réponse négative, s'ils parvenaient à analyser leur SLS pour procéder à des inférences, en les motivant. À ce stade, de nombreuses réponses de locuteurs apprenants consistaient à expliquer que TEMPÊTE DANS UN VERRE D'EAU dénote un problème rencontré. En effet, tout comme pour COUPER LES CHEVEUX EN QUATRE discuté en 2.2.2, l'image d'une perturbation qui se déchaîne dans un petite quantité d'eau destinée à être bue est suffisamment riche pour donner lieu à plus d'une métaphore, entre autres celle qui en fait un obstacle empêchant d'avaler le contenu du verre. Les contenus sémantiques de la SLS ont donc bel et bien été analysés, sans que l'on puisse qualifier la locution de véritablement transparente pour nos apprenants. L'hypothèse formulée sur le sens dénotatif de la locution fut erronée, et la motivation établie non pertinente. Comme le souligne Langlotz (2006), la motivation n'est pas construite par le locuteur au hasard, car elle repose toujours sur un nombre limité de métaphores (et - ajoutons - de métonymies) possibles, mais il se peut que l'on ne choisisse pas celle qui est réellement encodée en L2.

Pour la deuxième étape du test, un corpus d'exemples a été distribué aux étudiants : quinze extraits en provenance de la base textuelle Frantext illustraient les emplois des locutions précédemment soumises à l'analyse. Pour TEMPÊTE DANS UN VERRE D'EAU, nous avons sélectionné le passage suivant ${ }^{17}$ :

8. Mais j'avais tenu au courant votre état-major impérial. Nous avons une petite tempête dans un verre d'eau. Cela va s'apaiser très vite. (Frantext) 
Malgré le verbe S'APAISER dans le cotexte droit, cet exemple (comme la plupart de ceux que nous avons soumis aux deux groupes) a été jugé peu utile pour orienter les hypothèses sur le sens de la locution. Ce résultat peut s'expliquer par le caractère sémantiquement condensé mis en avant par Fellbaum, que nous avons déjà mentionné dans la section 2.2.1 ci-dessus : une locution permet de désigner, de manière courte et synthétique, des situations par ailleurs complexes. Une locution offre ainsi un condensé sémantique, qui permet des économies d'indices cotextuels. De plus, observons que le choix d'une locution telle que TEMPÊTE DANS UN VERRE D'EAU permet d'ajouter une attitude propositionnelle, en l'occurrence un jugement, qualifiant le désaccord d'inimportant.

\subsection{Locutions et compétences productives de l'apprenant}

Pour transmettre un contenu sémantique à l'aide d'une locution, l'apprenant doit récupérer dans son stock lexical sa forme complète, c'est-à-dire sa structure lexico-syntaxique (SLS). Il n'est pas possible, en effet, de l'assembler en fonction du sens à transmettre - c'est à ce stade donc qu'il faut composer avec le figement du signifiant locutionnel.

Lorsque l'apprenant souhaite dire par exemple qu'un individu $\mathrm{X}$ réalise des efforts en collaboration avec Y en vue d'objectifs communs, il lui est certes possible d'employer le verbe COLLABORER ou COOPÉRER, ou encore de recourir à un syntagme libre et compositionnel comme TRAVAILLER ENSEMBLE, etc. Mais le français met aussi à disposition des locuteurs la locution RAMER DANS LE MÊME SENS. Le figement du signifiant se manifestant notamment dans le blocage de substitution paradigmatique (cf. Gross 1996) représente alors un défi certain. L'exemple 9 ci-dessous, produit par un locuteur étranger, illustre le cas d'infraction à ce blockage :

9. Ici, personne ne rame dans la même direction que moi.

(Conversation privée, sans jeu de mots, mars 2015)

En produisant la phrase (9), le locuteur a remplacé SENS par DIRECTION ; les deux quasisynonymes étant en effet substituables dans de nombreux cotextes. Même si le signifié de SENS ne figure pas dans la définition de la locution (entièrement non-compositionnelle), il n'en joue pas moins un rôle pour ce qui est de son analysabilité et, par conséquent, pour la motivation du sens dénotatif. $\mathrm{Si}$, ayant auparavant rencontré et analysé cette locution via sa SLS, l'étudiant est parvenu à identifier la composante imagée et à en inférer le sens global, il n'est pas étonnant que les relations paradigmatiques d'un lexème tel que SENS restent actives $^{18}$. D'autant plus que, dans bien des cas, le blocage paradigmatique n'est pas complet : certaines locutions - par exemple MANGER DES YEUX - autorisent le recours aux quasi-synonymes, le cas échéant au verbe DÉVORER.

Les recherches menées sur 70 langues d'Europe et d'autres continents dans le cadre du projet Widespread Idioms in Europe and Beyond (Piirainen, 2008b) montrent que des locutions similaires sont largement attestées dans plusieurs langues de familles différentes : c'est le cas de NAGER CONTRE LE COURANT, par exemple. Cependant, ces équivalences doivent être observées avec une certaine prudence, car si ces ressemblances peuvent faciliter la démarche interprétative, elles constituent des sources de confusions potentielles dans la production des énoncés. Tout d'abord, même lorsqu'une locution est construite en L2 sur un patron syntaxique similaire, sa SLS peut être légèrement différente sur le plan lexical. Si dans plusieurs langues, un non-conformiste nage contre le courant, il peut également avoir à affronter une marée (en écossais, en portugais) ou une chute d'eau (en celte d'Irlande).

Ensuite, l'existence d'un équivalent locutionnel en L2 ne permet pas de conclure à l'identité sémantique. Dobrovol'skij (2016) analyse entre autres le cas de SCHWARTZ AUF 
WЕIß en allemand et celui de ЧЁРНЫМ ПО БЕЛОМУ en russe, également traduits en français par NOIR SUR BLANC. Or, bien que la composante imagée d'un texte imprimée de manière claire et nette soit comparable, le sens lexicalisé par la locution dans ces langues diffère sensiblement. Si le russe (comme le français) met en avant le caractère évident de l'information transmise, l'allemand insiste sur son côté officiel lié à l'impression d'un document. Une certaine méfiance s'impose donc face aux locutions en provenance de langues différentes, même là où leurs SLS présentent des similarités importantes, particularités flexionnelles mises à part.

Quand un locuteur étranger choisit d'exprimer un sens par une locution plutôt que par un lexème, il lui est nécessaire de s'assurer que la composante imagée est bien celle qu'il veut faire apparaître. Il n'est pas rare que l'apprenant souhaite transmettre à son interlocuteur celle qui se trouve lexicalisée dans sa L1, soit parce qu'il ne connaît pas de locution équivalente en L2, soit parce qu'il n'y trouve pas la même composante imagée. Ainsi, face à un excès de soins et d'attentions qui aboutissent à un résultat contraire de celui qui était escompté, un locuteur polonophone pourra employer l'expression ZAGŁASKAĆ KOTA NA ŚMIERĆ (lit. 'caresser le chat jusqu'à ce qu'il en crève'). Désireux d'en rendre le sens avec la composante imagée, il enchâsse une traduction mot à mot de la SLS dans une mise en garde de type "Comme on dit chez nous... ».

Ainsi, de quel côté que l'on se tourne, les difficultés se multiplient. Les propriétés structurelles et fonctionnelles des locutions constituent une source de problèmes considérables que les apprenants de L2 doivent savoir traiter et résoudre. D'une part, lors des premières rencontres dans le discours, elles imposent une démarche interprétative reposant sur une analyse et une recherche de liens entre le sens dénotatif, même lorsque celui-ci est entièrement non-compositionnel, et les lexèmes de sa SLS. De l'autre, leur emploi en discours ne peut se faire sans un traitement complexe ${ }^{19}$, dont il convient de tenir compte dans une démarche phraséodidactique.

\section{Démarches pédagogiques à construire dans la pratique de classe de langue}

Les propriétés spécifiques des locutions que nous venons de passer en revue et qui constituent des sources de problèmes importants dans l'apprentissage du vocabulaire doivent, bien évidemment, être prises en compte dans l'élaboration des cursus et dans la conception des activités de classe. Or, les formations en langue sont également soumises à d'autres contraintes. Nous ne nous arrêterons pas ici sur le manque de temps, qui pèse lourdement sur les choix pédagogiques, pour réfléchir à celles qu'impose une approche fortement utilitaire des méthodes actionnelles récommandées notamment par le Cadre européen de référence pour les langues (2001, North et Goodier 2018). Sans mettre en cause les méthodes actionnelles elles-mêmes, c'est la vision exclusive de l'apprenant « acteur social» promue par ces documents qui nous paraît pour le moins discutable (cf. Sikora 2016a) ${ }^{20}$. Nous convenons volontiers cependant de leur utilité pour l'organisation des cursus en ce qui concerne le rythme de développement des compétences et surtout, l'évaluation de leur acquisition. C'est d'ailleurs cet aspect pratique qui nous paraît le plus intéressant pour la phraséodidactique. Réinterprétés par les linguistes didacticiens, le CECRL ainsi que ses documents satellites ${ }^{21}$ fournissent un référentiel de difficultés à envisager et des indices pour introduire progressivement dans les apprentissages des locutions abordables à tel ou tel niveau de compétences. 


\subsection{Compétences d'apprenants selon le Cadre européen commun de référence pour les langues (CECRL)}

Dans la pratique de classe, l'utilité du CECRL tient aux niveaux de compétences qu'il définit et qui permettent de construire des programmes assurant une progression. La relative cohérence des apprentissages quel que soit leur cadre institutionnel est d'autant plus importante que dans leur immense majorité, les cours sont aujourd'hui orientés vers la valorisation des acquis par des certifications. Ainsi, en partant d'un niveau Pré-A1 (débutant complet), l'apprenant doit développer successivement un certain nombre d'aptitudes, en acquérant des connaissances linguistiques et socioculturelles. Au fur et à mesure que ses apprentissages progressent, il sera en mesure de réaliser de plus en plus de «tâches». Concernant l'étendue de vocabulaire d'un étudiant A2, les nouveaux descripteurs du CECRL stipulent que celui-ci :

Peut produire de brèves expressions courantes afin de répondre à des besoins simples de type concret : détails personnels, routines quotidiennes, désirs et besoins, demandes d'information.

Peut utiliser des modèles de phrases élémentaires et communiquer à l'aide de d'expressions mémorisées, de groupes de quelques mots et d'expressions toutes faites, sur soi, les gens, ce qu'ils font, les lieux, les biens, etc.

Possède un répertoire limité de courtes expressions mémorisées couvrant les premières nécessités vitales des situations prévisibles; des pannes fréquentes et des malentendus surviennent dans les situations imprévues. (North et Goodier $2018: 137)$

Un étudiant A2 doit donc être en mesure de parler de ses occupations quotidiennes, de préparer un voyage, d'apporter des informations sur ses goûts, ses loisirs et ses activités professionnelles par exemple. Parmi les tâches qu'il doit pouvoir réaliser figurent par exemple l'achat d'un billet, la réservation d'un hôtel ou la prise d'un rendez-vous, à l'aide - conformément à ses compétences grammaticales - de structures simples. Avec ce bagage lexical, l'apprenant «[p]eut utiliser des structures simples correctement mais commet encore systématiquement des erreurs élémentaires comme, par exemple, la confusion des temps et l'oubli de l'accord ; le sens général reste cependant clair » (ibidem, p. 139). Toutes les compétences sont ensuite progressivement développées jusqu'à un niveau $\mathrm{C} 2$ de locuteur autonome.

Les expressions idiomatiques parmi lesquelles comptent les locutions sont mentionnées seulement parmi les compétences du niveau C1. L'étudiant peut alors «comprendre un vaste éventail d'expressions idiomatiques, variations de registres et ajuster son propre registre » (ibidem, p. 144). Cependant, conformément à ces mêmes descripteurs, une bonne maîtrise de ces UP, reposant notamment sur une claire conscience des différents niveaux connotatifs, n'est attendue qu'en C2.

Les propriétés structurelles, sémantiques et fonctionnelles des locutions que nous avons discutées dans les sections précédentes, avec le lot de difficultés qu'elles occasionnent d'une part, les descripteurs de compétences du CECRL de l'autre pourraient conduire à la conclusion selon laquelle l'intégration de ces phrasèmes dans les apprentissages n'est pas à envisager avant le niveau C1. Dans les faits, cela signifierait que ce volet phraséodidactique n'est plus vraiment didactique, puisqu'à ce stade, l'apprenant est quasi autonome et n'a plus besoin d'une démarche guidée.

Néanmoins, une lecture attentive des descripteurs de compétences prévues en A2 (supra) indique à notre avis le contraire. HÔTESSE DE L'AIR, SOLDAT DU FEU, SALLE À MANGER ou encore ROUGE À LĖVRES sont bien des expressions courtes aux structures syntaxiques non seulement simples, mais aussi récurrentes en français. Faciles à mémoriser, elles permettent de parler des routines et des situations quotidiennes. Il est donc 
bien possible, et à nos yeux souhaitable, d'enseigner le vocabulaire locutionnel à ce niveau peu avancé, tout en respectant la progression dans le développement des compétences, à condition cependant d'adopter des pratiques réfléchies et ciblées.

\subsection{Locutions et phraséodidactique dans un cursus de langue}

De nombreuses études (Bogaards 1994, Meara et Wolter 2004, Milton 2009 à titre d'exemple) montrent que lorsqu'une démarche pédagogique cible un ensemble d'unités lexicales, celles-ci sont acquises de manière plus efficace et durable qu'en cas de rencontres aléatoires dans des textes ou dans des échanges quotidiens. D'un point de vue quantitatif, la différence est notable : selon les activités menées en classe, le nombre de mots appris par heure d'exposition peut aller du simple au triple (cf. Milton 2009). Compte tenu des propriétés structurelles et fonctionnelles qui caractérisent les locutions et de l'ensemble de difficultés qu'elles présentent, il apparaît d'autant plus important d'adopter une démarche pédagogique appropriée, construite à partir d'une conceptualisation de ces unités lexicales. Leur fréquence peu élevée (Colson 2008) en corpus est un argument supplémentaire en faveur d'une phraséodidactique raisonnée: ayant peu de chances de rencontrer régulièrement une même locution dans le discours dans plusieurs contextes différents, l'apprenant a d'autant plus besoin d'acquérir des connaissances et des stratégies nécessaires pour l'intégrer dans son vocabulaire.

\subsubsection{Place et objectifs de la phraséodidactique}

La phraséodidactique n'est pas une démarche à part: elle doit au contraire s'intéger à l'ensemble des activités de classe, en en partageant les finalités et le rythme de progression. Elle poursuit néanmoins deux types d'objectifs qui lui sont propres.

Comme pour tout enseignement lexical, son objectif premier est de développer le vocabulaire de l'apprenant d'un point de vue quantitatif. L'étudiant a besoin de maîtriser des unités du lexique, et celles-ci ne sont pas toujours des lexèmes. C'est ainsi qu'il élargit progressivement le nombre de domaines (cf. CECRL) dont il peut parler et la granularité des informations qu'il est en mesure de comprendre et de transmettre. Par exemple, pour traiter, à l'oral comme à l'écrit, des questions gastronomiques, il lui sera possible d'aller au-delà de PAIN, BEURRE, VIANDE, TOMATE, etc. avec des locutions telles que ASSIETTE DU TERROIR, MISE EN BOUCHE, CIGARETTE RUSSE, PIÈCE MONTÉE, POMME FRITE et, bien évidemment, PLAISIRS DE LA TABLE ${ }^{22}$ : le vocabulaire du champ sémantique de 'nourriture' en devient plus riche, plus diversifié, plus chargé culturellement.

Le second objectif pourrait être qualifié de stratégique: en plus d'apprendre une à une un certain nombre de locutions, il s'agit de mettre progressivement en place un modus operandi. En effet, le lexique d'une langue telle que le français offre une multitude de phrasèmes non-compositionnels, tout en s'enrichissant continuellement de nouvelles unités phraséologiques, y compris locutionnelles. On pourrait difficilement imaginer un parcours de L2 entièrement orienté vers la phraséodidactique, et quand bien même cela serait possible, il est peu probable qu'il parvienne à en faire un tour complet. En tant que locuteur, l'apprenant doit donc acquérir des stratégies qui lui permettent de traiter et de maîtriser en autonomie les locutions qu'il rencontre. Cyr (1998:5) indique à juste titre que «l'apprentissage d'une L2 peut être vu comme tout autre processus de traitement de l'information ». Or, dans le cas des locutions, ces informations sont, comme nous l'avons vu dans les sections précédentes, très complexes. Pour les traiter, l'apprenant a besoin de stratégies appropriées, c'est-à-dire de procédures et d'outils à mettre en œuvre pour améliorer ses apprentissages (cf. définition de statégie proposée par Oxford 1990).

L'une des stratégies les plus fréquentes consiste à consulter une ressource dictionnairique. Cette stratégie peut, du moins partiellement, apporter le résultat escompté, 
si l'information recherchée s'y trouve. Mais sa mise en place repose sur une autre démarche, à savoir celle qui consiste à repérer dans le texte une unité de sens au signifiant polylexémique. Autrement dit, l'apprenant doit avoir conscience qu'un sens dénotatif à prendre en compte dans le calcul compositionnel peut être porté par un phrasème, c'est-àdire par une forme syntagmatique. La question est donc de savoir comment, sans se lancer dans un cours de linguistique et de lexicologie, simplement à travers des activités de classe, apprendre à apprendre le vocabulaire locutionnel.

\subsubsection{Locutions dans l'enseignement de vocabulaire}

Notre objectif dans cet article n'est pas de proposer une séquence pédagogique consacrée à l'apprentissage de locutions. La pratique de classe montre qu'il est difficile de construire un cours - un ensemble d'activités structurées qui tendent vers des objectifs bien déterminés sans penser aux apprenants que l'on aura en face de nous. Ainsi, nous réunissons ci-dessous des éléments dont - à notre avis - il convient de tenir compte, si l'on veut concevoir une didactique du lexique incluant le vocabulaire locutionnel. Même si la phraséodidactique ne limite pas son champ d'action aux locutions, nous nous en tiendrons ici strictement à ces phrasèmes, car il s'agit de réfléchir à une pédagogie qui prenne en compte les propriétés formelles et fonctionnelles des unités phraséologiques d'un type particulier. Des propositions pédagogiques portant sur les locutions peuvent s'avérer inapplicables aux collocations ou aux pragmatèmes du fait de leurs caractéristiques différentes.

Malgré la complexité de leurs structures syntaxiques et sémantiques, il nous semble essentiel d'introduire des unités locutionnelles dès le niveau A2, voire A1, à condition néanmoins de les choisir soigneusement. Les critères à appliquer sont multiples : il est possible notamment de les sélectionner en tenant compte de leur complexité formelle et sémantique d'une part, et des récommandations du CECRL de l'autre. Si nous les discutons ci-dessous sous forme d'une liste, il ne faut en aucun cas y chercher une hiérarchie à respecter. En réalité, il s'agit de critères que l'on doit croiser.

1. Les domaines définis dans le CECRL correspondent grosso modo à ce que la linguistique qualifie de champs sémantiques. En A1, il s'agit de privilégier le vocabulaire relatif à la vie de famille, aux professions, à la nourriture, ainsi qu'à l'environnement matériel immédiat (objets quotidiens, meubles, accessoires, etc.). L'objectif à ce stade des apprentissages est de nommer des objets, plutôt que de les qualifier ou de s'intéresser aux relations entre eux. Il est dès lors possible de proposer un travail autour des locutions telles que HÔTESSE DE L'AIR, SOLDAT DU FEU, FER À REPASSER, MACHINE À LAVER, SALLE DE BAINS, PETIT COPAIN, etc. Deux bénéfices potentiels en découlent. En ce qui concerne le développement quantitatif du vocabulaire, les apprenants acquièrent des moyens de communiquer dans les champs sémantiques/domaines correspondant à leur niveau de compétences. Parallèlement, ils sont amenés vers des stratégies appropriées notamment vers une prise de conscience qu'un signifié n'est pas nécessairement associé à un lexème isolé: son signifiant est souvent polylexical.

2. Le travail avec un public débutant est orienté vers le concret, comme le montrent les domaines/champs sémantiques détaillés dans le CECRL. Il est donc préférable de se concentrer sur des locutions comme celles que nous venons d'évoquer, dont la description sémantique repose essentiellement sur le sens dénotatif, sans (trop de) recours à une composante imagée (cf. sous-section 2.2 supra). Au fur et à mesure que l'étudiant progresse vers des niveaux plus avancés, il est possible de prendre en charge les contenus figuratifs, en concevant des activités autour des images sous-jacentes des sens dénotatifs. 
3. Si l'on s'en remet au descriptif des compétences proposé dans le CECRL, il convient également de tenir compte des propriétés structurelles du signifiant. Les étudiants A1 et A2 sont en mesure de s'exprimer avec des constructions simples. Par ailleurs, le vocabulaire nominal semble plus accessible pour les apprenants à cette étape (cf. la sous-section 2.1.3 supra). Tous ces indices conduisent à opter, dans un premier temps, pour des locutions nominales, avant d'introduire des phrasèmes locutionnels relevant des autres parties du discours.

4. Pour permettre aux étudiants A1 d'intégrer ces locutions dans leur vocabulaire productif, notamment à travers des exercices et activités de réemploi, il est nécessaire de s'assurer que la partie du discours de surface correspond aux fonctions que la locution remplit dans la phrase, c'est-à-dire à sa partie du discours profonde (cf. sous-section 2.1.3 supra). Conformément aux récommandations du CECRL, en $\mathrm{A} 1$, le locuteur apprenant recourt à des phrases simples de forme Sujet Verbe Complément. Le maniement de syntagmes nominaux qui s'emploient non pas comme des sujets ou des compléments, mais comme des modificateurs adverbiaux et/ou adjectivaux, pourra difficilement être envisagé avant un niveau de compétences B2.

5. À des niveaux avancés, il est important de proposer aux étudiants un travail de classe visant à la fois le sens dénotatif et son élaboration à partir de la composante imagée qu'il contient. Autrement dit, il s'agit d'accompagner les apprenants dans leur recherche de la motivation d'une locution pour les aider à identifier ces contenus figuratifs. Plusieurs raisons justifient un tel choix pédagogique. D'un point de vue linguistique, on ne peut pas employer une locution dans le discours sans maîtriser l'image qu'elle véhicule. Pour l'acquisition, conformément à la théorie de double codage (dual coding theory) développée par Clark et Paivio (1991), la mise en relation des informations lexicales et des contenus figuratifs faciliterait la mémorisation et le ré-emploi en contexte.

Les critères énumérés ci-dessus permettent de sélectionner un ensemble de locutions en accord avec le niveau de compétences des apprenants, avec un degré de difficulté qui n'est pas insurmontable pour le public concerné. Elles peuvent être intégrées dans les compétences réceptives et productives.

Les méthodes communicatives et actionnelles privilégient néanmoins l'usage de documents authentiques, originellement destinés aux locuteurs natifs. Si l'enseignant est parfois amené à les adapter à son public (par exemple en les raccourcissant), il n'est pas toujours possible, ni souhaitable, de les expurger complètement de tout élément idiosyncrasique. On peut alors y trouver une locution qui présente un niveau de difficulté trop élevé pour le niveau de compétences des apprenants. C'est à l'enseignant qu'il revient alors de faire un diagnostic et d'adopter une démarche appropriée, mais il lui faudra pour cela une connaissance des différentes facettes des unités du lexique et des problèmes qu'elles occasionnent. S'il juge une locution trop complexe sur le plan formel, sémantique et combinatoire pour s'attendre à ce qu'elle soit réemployée par l'apprenant, peut-être pourra-t-il limiter ses explications au sens dénotatif, en laissant (temporairement) de côté les contenus figuratifs ? Avec des apprenants plus avancés, au contraire, il sera possible d'organiser des activités ciblant justement la composante imagée, pourquoi pas dans une perspective comparée plurilingue dans des classes hétéroglottes? L'enseignement n'est-il pas l'art de choisir les informations pertinentes pour soutenir les apprentissages hic et nunc, tout en préparant le terrain pour ceux à venir? 


\section{Conclusion}

L'objectif que nous nous sommes posé en entamant cette réflexion était de montrer en quoi une approche lexicologique des locutions devrait permettre d'envisager une démarche phraséodidactique dans l'enseignement de vocabulaire en L2. Certes, la phraséodidactique ne se limite aucunement aux locutions fortes, mais c'est à dessein que cette étude s'est focalisée sur ce type de phrasèmes. Il s'agissait de montrer comment leur conceptualisation dans un modèle lexical conduit à en penser une approche pédagogique. Pour ce faire, dans la section 2, nous avons étudié leurs propriétés formelles, sémantiques bien décrites par la lexicologie, afin de vérifier en quoi ces caractéristiques représentent des sources de difficulté potentielles pour les apprenants. Nous avons discuté ensuite (section 3), l'articulation entre ce que nous avons qualifié de propriétés fonctionnelles et les deux types de compétences - réceptives et productives. En réunissant les éléments ainsi dégagés, nous venons d'esquisser, dans la section 4 , les grandes lignes pour un traitement pédagogique des locutions.

Faute de place, le présent article s'arrête à une étape où il serait intéressant de rebrousser chemin et d'envisager les rapports entre la lexicologie et la lexicographie d'une part, et la (phraséo)didactique de l'autre selon une perspective inverse. Nous pensons en effet que tout comme l'étude du lexique et la réflexion sur les entités qui le constituent aident les enseignants à concevoir des parcours d'apprentissage, une conscience claire des besoins des utilisateurs enseignants et apprenants permet aux lexicologues et aux lexicographes d'améliorer la macro- et la microstructure des ressources lexicales, en y incluant des informations necessaires et en les structurant de manière à guider les uns et les autres. Présenter une unité phraséologique en tant qu'entrée lexicographique à part entière, avec un article qui la décrit devient une pratique courante (cf. The Cambridge English Dictionary en ligne, les bases WordNet de différentes langues ou les réseaux lexicaux comme le $R L-f r$ ), sans pour autant être devenu la règle. Certaines ressources fournissent, dans les articles dictionnairiques, des informations très utiles pour la pratique pédagogique. Par exemple, le système d'étiquettes sémantiques (cf. Polguère 2011) implémenté dans les réseaux lexicaux tels que le $R L-f r$ permettra à terme l'extraction d'unités lexicales relevant d'un même champ sémantique.

De plus, les dictionnaires fournissent parfois des indications précises (et précieuses !) quant aux niveaux de compétences d'apprenants susceptibles d'en acquérir la maitrise. The Cambridge English Dictionary par exemple estampille la locution DOWN IN THE DUMPS de la marque $\mathrm{C} 2$, en précisant ainsi à quel niveau de compétences elle correspond. Autrement dit, les lexicologues indiquent explicitement que ce phrasème est à étudier par des apprenants $\mathrm{C} 2$, qui sont déjà des locuteurs autonomes.

Les échanges entre les linguistes - lexicologues et lexicographes tout particulièrement pour ce qui concerne les enseignements lexicaux - et les acteurs du terrain (enseignants, didacticiens, apprenants eux-mêmes) offrent de nouvelles perspectives à tous ceux qui y participent. Nous espérons avoir montré ce que la lexicologie peut apporter à l'enseignement: une modélisation des données lexicales et une conceptualisation des entités qui le forment. En adossant ses activités à un modèle robuste, l'enseignant se dote d'outils permettant de proposer des parcours d'apprentissage structurés, en anticipant les difficultés. Nous n'avons fait que signaler rapidement, en attendant de développer ces idées ailleurs, que les bénéfices sont réciproques, car la pratique de classe et la réflexion didactique indiquent en retour comment enrichir les informations lexicales proposées dans les ressources dictionnairiques. 


\section{Bibliographie}

Aitchison, J. (2003). Words in the Mind. An Introduction to the Mental Lexicon. Oxford: Basil Blackwell.

Allen, E. D. et Valette, R. (1972). Modern Language Classroom Techniques: A Handbook. New York: Harcourt, Brace, Jovanovich.

Berthemet, E. (2017). Manuels et apprentissage du lexique idiomatique. $85^{\mathrm{e}}$ Congrès de l'Association Francophone pour le Savoir (ACFAS), Université McGill, Montréal, Canada, 8-12 mai 2017.

Bogaards, P. (1994). Le vocabulaire dans l'apprentissage des langues. Paris: Hatier/Didier.

Cadre européen commun de référence pour les langues. Apprendre, évaluer, enseigner. (2001). Conseil de l'Europe. <en ligne> rm.coe.int/16802fc3a8, consulté le 20 mars 2018.

Clark, J. M. et Paivio, A. (1991). Dual coding theory and education. Educational Psychology Review, 3, 233-262.

Colson, J.-P. (2008). Cross-linguistic phraseological studies. Dans S. Granger et F. Meunier (éds). Phraseology. An Interdisciplinary Perspective. Amsterdam, Philadelphia (Pa): John Benjamins Publishing Company, 191-206.

Cuq, J.-P. (2004). Le lexique en situation d'apprentissage guidé : pour une méthodologie d'enseignement interventionniste dans l'enseignement du français langue étrangère. Dans É. Calaque et J. David (éds), Didactique du lexique. Contextes, démarches, supports. Bruxelles: De Boeck, 61-71.

Cyr, P. (1998). Les stratégies d'apprentissage. Paris : Clé-international.

Dobrovol'skij, D. (2016). The notion of "inner form" and idiom semantics. Études et travaux d'Eur'ORBEM, 1, 21-36.

Dobrovol'skij, D. et Piirainen, E. (2005). Figurative Language. Cross-Cultural and Cross-Linguistic Perspectives. Amsterdam, Boston: Elsevier.

Gibbs, R. W. J. (1994). The Poetics of Mind. Figurative Thought, Language, and Understanding. Cambridge, New York: Cambridge University Press.

Gläser, R. (1988). The grading of idiomaticity as a presupposition for a taxonomy of idioms. Dans W. Hüllen et R. Schulze (eds), Understanding the Lexicon. Tübingen: Max Niemeyer, 264-279.

Gréciano, G. (1994). Vorsicht, Phraseoaktovität! Dans B. Sandig (ed.), Tendenzen der Phraseologieforschung. Universitätsverlag Brockmeyer.

Gross, G. (1996). Les expressions figées en français. Noms composés et autres locutions. Paris : Ophrys.

Kleiber, G. (1990). La sémantique du prototype. Paris : Presses universitaires de France.

Kleiber, G. (1999). Problèmes de sémantique. La polysémie en questions. Villeneuve d'Asq: Éditions du Septentrion.

Klein, W. (1995). Second Language Acquisition. Cambridge. Cambridge: Cambridge University Press.

Langlotz, A. (2006). Idiomatic Creativity. A Cognitive-Linguistics Model of Idiom Representation and Idiom-Variation in English. Amsterdam, New York: John Benjamins Publishing Company.

Laufer, B. (1990). Why are some words more difficult? - Some intralexical factor that affect the learning of words. International Review of Applied Linguistics in Language Teaching, 28(4), 293-308.

Lewis, M. (1993). The Lexical Approach. The State of ELT and a Way Forward. London: Language Teaching Publications.

Lewis, M. (2008). Implementing the Lexical Approach. Putting Theory into Practice. Cheriton: Heinle.

Meara, P. (2009). Connected words: Word associations and second language vocabulary acquisition. Amsterdam, Philadelphia (Pa): John Benjamins.

Meara, P. et Wolter, B. (2004). V_Links: Beyond Vocabulary Depth. Angles on the English Speaking World, 4, 85-97.

Mel'čuk, I. (2013). Tout ce que nous voulions savoir sur les phrasèmes, mais... Cahiers de Lexicologie, 102 (1), 129-149.

Mel'čuk, I., Clas, A. et Polguère, A. (1995). Introduction à la lexicologie explicative et combinatoire. Bruxelles : De Boeck Supérieur.

Milton, J. (2009). Measuring Second Language Acquisition. Bristol: Multilingual Matters.

Nation, I. P. A. (1982). Beginning to learn foreign language vocabulary. RELC Journal, 13, 61-68.

Nation, I. P. A. (1990). Teaching and learning vocabulary. New York: Newbury House. 
North, B., Goodier, T. (2018). Cadre européen de référence pour les langues: apprendre, enseigner, évaluer. Volume complémentaire avec les nouveaux descripteurs. Strasbourg: Conseil de l'Europe. $\quad<$ en ligne > rm.coe.int/cecr-volume-complementaire-avec-de-nouveauxdescripteurs/16807875d5, consulté le 20 mars 2018.

Oxford, R. (1990). Language Learning Strategies: What Every Teacher Should Know. New York: Newbury House Publishers.

Pausé, M.-S. (2017). Structure lexico-syntaxique des locutions du français et incidence sur leur combinatoire. Université de Lorraine et ATILF CNRS (UMR 7118), Nancy.

Phillips, T. A. (1981). Difficulties in Foreign Language Vocabulary Learning and a Study of Some Factors Thought to be Influential. M.A. Project, Birkbeck College, University of London.

Piirainen, E. (2008a). Figurative phraseology and culture. Dans S. Granger et F. Meunier (eds), Phraseology. An Interdisciplinary Perspective. Amsterdam, Philadelphia (Pa): John Benjamins Publishing Company, 207-228.

Piirainen, E. (2008b). Phraseology in a European framework. A cross-linguistic and cross-cultural project on widespead idioms. Dans S. Granger et F. Meunier (eds), Phraseology. An interdisciplinary perspective. Amsterdam, Philadelphia $(\mathrm{Pa})$ : John Benjamins Publishing Company, 243-258.

Polguère, A. (2011). Classification sémantique des lexies fondée sur le paraphrasage. Cahiers de Lexicologie, 98 (1), 197-211.

Polguère, A. (2014). From Writing Dictionaries to Weaving Lexical Network. International Journal of Lexicography, 27 (4), 396-418.

Polguère, A. (2016). La question de la géométrie du lexique. Dans F. Neveu, G. Bergounioux, M.-H. Côté, J.-M. Fournier, L. Hriba, et S. Prévost (éds.), Actes $d u$ " $5 e$ Congrès Mondial de Linguistique Française (CMLF 2016)». Université de Tours, <en ligne> dx.doi.org/10.1051/shsconf/20162701002

Rodgers, T. S. (1969). On measuring vocabulary difficulty: an analysis of item variables in learning Russian-English vocabulary pairs. International Review of Applied Linguistics, 7, 327-343.

Rosch, E. (1975). Cognitive Representation of Semantic Categories. Journal of Experimental Psychology, 104, 192-233.

Schur, E. (2007). Insights into the structure of L1 and L2 vocabulary networks: intimtions of small worlds. Dans H. Daller, J. Milton, et J. Treffers-Daller (eds.), Modelling and Assessing Vocabulary Knowledge. Cambridge, New York: Cambridge University Press, 182-203.

Sikora, D. (2016a). Activité(s) de définition dans l'apprentissage d'une langue seconde. Cahiers de Lexicologie, 2 (109), 117-143.

Sikora, D. (2016b). Identification d'unités phraséologiques et équivalence sémantique dans la traduction. In Computerised and Corpus-based Approaches to Phraseology: Monolingual and Multilingual Perspectives/Fraseología computacional y basada en corpus: perspectivas monolingües $y \quad$ multilingües. Genève: Tradulex. <en ligne> www.tradulex.com/varia/Europhras2015.pdf,

Svensson, M. H. (2008). A very complex criterion of fixedness: Non-compositionality. Dans S. Granger et F. Meunier (eds), Phraseology. An Interdisciplinary Perspective. Amsterdam, Philadelphia (Pa): John Benjamins Publishing Company, 81-93.

Taylor, J. R. (1995). Linguistic Categorization. Oxford: Oxford University Press.

${ }^{1}$ Je remercie chaleureusement Gaétane Dostie et les relecteurs anonymes : leur regard attentif et leurs remarques, questions et propositions d'amélioration m'ont beaucoup aidée dans la préparation de la version finale de cet article.

${ }^{2}$ Nous parlerons indifféremment de langue étrangère, français langue étrangère (FLE) et de langue seconde L2, en admettant avec Klein (1995) que toute langue apprise après une première est seconde. Pour cet exposé, il n'est pas indispensable de procéder à des distinctions plus fines entre une L2 et une L3.

${ }^{3}$ Tous mes remerciements au relecteur anonyme pour avoir attiré mon attention sur ce cas de figure, qui est effectivement à distinguer de celui où l'apprenant doit lui-même décider de la forme fléchie à adopter. 
${ }^{4}$ Les similarités de structures en réseau ne signifient pas l'identité. Pour une présentation et discussion des différences dans l'organisation du vocabulaire chez un locuteur de L1 et chez un apprenant, voir Schur (2007).

${ }^{5}$ La locution AUX ANGES a été discutée dans Sikora (2016b) en rapport avec un problème plus large d'identification des structures et, par conséquent, de la nature des locutions. Les arguments réunis montrent qu'il s'agit en effet non pas d'une locution verbale ÊTRE AUX ANGES, mais d'une locution prépositionnelle à emplois adjectivaux. Elle peut en revanche constituer une base pour des collocations formées avec les verbes supports ÊTRE, SEMBLER et METTRE.

${ }^{6}$ Rappelons que le $R L-f r$ est une implémentation d'un modèle relationnel de type système lexical, qui prend en compte tous les liens (paradigmatiques, syntagmatiques, ainsi que ceux d'inclusion formelle), sans les hiérarchiser (cf. Polguère 2014, 2016). Un tel réseau de connexions paradigmatiques représente une description relativement riche du sens dénotatif, même en l'absence de paraphrase définitoire. Une exploration d'un sousréseau à la recherche d'informations sémantiques représente pour l'apprenant une tâche significative, c'est-à-dire l'impliquant personnellement. Or, comme l'observe Cuq (2004), ce caractère significatif des tâches réalisées en cours d'apprentissage est décisif pour son efficacité.

${ }^{7}$ Nous reprenons ainsi la définition de non-compositionnalité proposée par Mel'čuk (2013) sans pour autant en reproduire la formalisation, qui ne semble pas indispensable pour les problèmes qui nous occupent ici.

${ }^{8}$ Communication personnelle.

${ }^{9}$ Accessible via la plateforme du Centre National de Ressources Textuelles et Lexicales (www.cnrtl.fr).

${ }^{10} \mathrm{Cf}$. Mel'čuk et al. (1995: 60)

${ }^{11}$ Notons que le TLFi, consulté via la plateforme CNRTL mentionne pour le français un emploi de figuratif au sens de figuré (cf. www.cnrtl.fr/definition/figuratif), acception qui semble d'ailleurs première du point de vue diachronique.

${ }^{12}$ Cf. Dobrovol'skij (2016 : 23) : "the image component (...) of a figurative lexical unit is defined as the linguistically relevant traces of the mental image underlying its semantics."

${ }^{13}$ Pausé (2017) parle à ce propos de projection structurale.

${ }^{14}$ Nous n'utilisons pas ici le terme de cadre (cf. frame, Dobrovol'skij 2016) découpant un domaine source pour la métaphore que serait alors la locution. Comme nous l'avons expliqué plus haut, il est problématique - dans un modèle synchronique - d'analyser une locution (donc une unité lexicale) appartenant au lexique contemporain comme métaphore d'un syntagme libre qui n'a pas sa stabilité au sein du module lexical. Nous convenons que la présente explication est un peu expéditive et qu'elle mériterait une justification plus étoffée, difficile à fournir dans les limites de cet article.

${ }^{15}$ Chacun de ces deux types se subdivise en compétences relatives à l'écrit et à l'oral. Notons au passage que les compétences réceptives sont en général nettement plus étendues que les compétences productives.

${ }^{16}$ Il est préférable de parler de contenus sémantiques, plutôt que de sens, puisque parfois, la réanalyse effectuée s'appuie également des connotations, comme dans le cas de PEAU DE BANANE.

${ }^{17}$ Dans un sous-corpus de travail allant de 1950 à 2017 défini dans Frantext, seules six occurrences de cette locutions apparaissent. Nous avons sélectionné la concordance qui contenait une indication - S'APAISER - susceptible d'orienter les inférences.

${ }^{18}$ Rappelons que plusieurs travaux consacrés au lexique mental (cf. Aitchison 2003 pour une discussion) mettent en avant le rôle structurant des relations paradigmatiques dans son organisation. 
${ }^{19}$ Nous ne parlerons pas ici de flexibilité formelle (Pausé 2017) qui autorise par exemple la passivation ou l'introduction de modificateurs, pas plus que nous n'évoquerons les défigements. Ces faits de langue sont affaire de locuteurs natifs et autonomes d'une langue (niveaux $\mathrm{C} 1$ et $\mathrm{C} 2 \mathrm{du} \mathrm{CECRL}$ ). Compte tenu de leurs compétences, ces derniers ne sont plus à considérer comme des apprenants à proprement parler.

${ }^{20}$ Par rapport à la version de 2001 du CECRL, la lecture du volume complémentaire du CECRL, paru en février 2018 (North et Goodier 2018) permet de constater une certaine ouverture, notamment vers une réflexion sur la langue, et donc un retour réflexif et critque sur les connaissances la concernant. C'est dire que l'apprenant y apparaît non plus seulement comme destiné à réaliser des tâches essentiellement sociales et des projets au sein d'un groupe. On peut donc espérer qu'à terme il soit envisagé selon une dimension plus personnalisée et personnalisante en tant qu'acteur de ses propres apprentissages (cf. Sikora 2016a).

${ }^{21}$ Parmi les documents qui complètent le CECRL, citons à titre d'exemple l'outil d'autoévaluation intitulé Portfolio européen des langues (PEL).

${ }^{22}$ Ces locutions ont été extraites de la base $R L-f r$ via une requête portant sur l'étiquette sémantique 'aliment préparé'. Celle-ci a permis d'afficher toutes les lexies porteuses de cette étiquette. C'est dire qu'une ressource lexicale structurée de manière adéquate est un outil précieux dans le travail d'un enseignant. Nous y revenons dans la section 5, qui conclura nos réflexions. 